\title{
Early Relapse of Unresectable Gallbladder Cancer after Discontinuation of Gemcitabine Monotherapy Administered for 5 Years in a Patient Who Had Complete Response to the Treatment
}

\author{
Koichi Suyama ${ }^{a, c}$ Masafumi Ikeda ${ }^{a}$ Eiichiro Suzuki, d \\ Motohiro Kojima $^{b}$ Shuichi Mitsunaga ${ }^{a}$ Satoshi Shimizu ${ }^{a}$ Izumi Ohno $^{a}$ \\ Hideaki Takahashi $^{a} \quad$ Hiroyuki Okuyama $^{a}$ Akiko Kuwahara $^{a}$ \\ Takuji Okusaka ${ }^{\mathrm{e}}$ Junji Furuse $\mathrm{a}^{\mathrm{a}} \mathrm{f}$ \\ Divisions of ${ }^{\mathrm{a}} \mathrm{Hepatobiliary}$ and Pancreatic Oncology, and ${ }^{\mathrm{b}}$ Pathology, National Cancer \\ Center Hospital East, Kashiwa, 'Department of Medical Oncology, Toranomon Hospital, \\ Tokyo, ${ }^{d}$ Department of Gastroenterology and Neghrology, Chiba University, Chiba, \\ ${ }^{\mathrm{e}}$ Hepatobiliary and Pancreatic Oncology Division, National Cancer Center Hospital, and \\ fDepartment of Internal Medicine, Medical Oncology, Kyorin University School of \\ Medicine, Tokyo, Japan
}

\section{Key Words}

Gallbladder cancer $\cdot$ Gemcitabine $\cdot$ Chemotherapy $\cdot$ Complete response $\cdot$ Early relapse

\begin{abstract}
The tumor shrinkage effect of gemcitabine is considered to be limited in cases of advanced gallbladder cancer, and there are few reports of complete response to gemcitabine therapy in patients with this cancer. Therefore, the treatment continuation strategy in these patients, after a complete response has been achieved, still remains to be established. Here, we present the case of a 77-year-old patient with unresectable gallbladder cancer, who after showing complete response to gemcitabine monotherapy administered for 5 years, showed early relapse within only 11 months of discontinuation of the drug. Thus, it is necessary to
\end{abstract}


Suyama et al.: Early Relapse of Unresectable Gallbladder Cancer after Discontinuation of Gemcitabine Monotherapy Administered for 5 Years

establish a suitable treatment continuation strategy for patients who show complete response to gemcitabine treatment.

(c) 2013 S. Karger AG, Basel

\section{Introduction}

The incidence of biliary tract cancer, including that of gallbladder cancer, has been increasing in Japan over the past several decades. Surgical resection offers the only possibility of cure; however, despite the recent advances in diagnostic and therapeutic modalities, the cancer is frequently diagnosed at an advanced stage, and the vast majority of patients with gallbladder cancer are unsuitable candidates for curative surgical treatment. The prognosis of advanced gallbladder cancer remains extremely poor, with a median survival time of these patients of 6-12 months [1, 2]; furthermore, patients surviving for at least 5 years are extremely rare.

Gemcitabine is a novel nucleoside analogue that exerts its efficacy after being phosphorylated to its active metabolite in the body following administration. Gemcitabine triphosphate competes with deoxycytidine triphosphate for incorporation into DNA, thereby inhibiting DNA synthesis [3]. Gemcitabine monotherapy has been reported as one of the effective treatments in patients with advanced gallbladder cancer [4-6]; however, there are few reports of complete response (CR). Therefore, no suitable treatment continuation strategy has been established for patients with advanced gallbladder cancer showing CR to gemcitabine monotherapy. Here, we present the case of a patient with unresectable gallbladder cancer, who after showing CR to gemcitabine monotherapy administered for 5 years, showed relapse within only 11 months of discontinuation of the treatment.

\section{Case Presentation}

A 77-year-old man with a 2-month history of right shoulder and upper abdominal pain visited his physician at another hospital. A gallbladder tumor, about $3 \mathrm{~cm}$ in diameter, was detected by ultrasonography, and the patient was then referred to our hospital for further diagnosis and treatment. His laboratory findings at presentation were nearly normal, including the serum levels of tumor makers. Computed tomography (CT) and magnetic resonance imaging (MRI) of the abdomen showed the mass to be located at the cervix of the gallbladder and to involve the common and right hepatic arteries, with enlargement of the No. 12 lymph node (fig. 1). A percutaneous biopsy of the tumor revealed an adenocarcinoma, and immunohistochemical analysis of the tumor showed positive staining for carcinoembryonic antigen (CEA) and cytokeratin 7, and negative staining for cytokeratin 20 . Thus, the tumor was diagnosed as an unresectable gallbladder cancer.

The patient was initiated on treatment with gemcitabine at a dose of $1,000 \mathrm{mg} / \mathrm{m}^{2}$ administered on days 1,8 , and 15 of each 4 -weekly cycle. After 13 cycles of treatment, a marked decrease of the tumor size on CT was noted (fig. 2a), and the treatment response was rated as partial response according to the Response Evaluation Criteria in Solid Tumors (RECIST) criteria, version 1.0. After 15 cycles of treatment, the tumor disappeared altogether, and CR was confirmed by CT after 20 cycles of gemcitabine monotherapy (fig. 2b). After CR had been achieved, the tumor was reevaluated for possible surgical resection by our surgeon. However, the patient was judged to be an unsuitable candidate for surgery, because he had severe bronchial asthma. Therefore, gemcitabine monotherapy was continued. After 27 cycles of treatment, corresponding to a further 12 months of treatment after CR had been 
Suyama et al.: Early Relapse of Unresectable Gallbladder Cancer after Discontinuation of Gemcitabine Monotherapy Administered for 5 Years

confirmed, we held a discussion with the patient and his family about whether or not they would like the gemcitabine therapy to be continued. They made the decision for the gemcitabine therapy to be continued, for fear that the tumor might relapse. At this time, because of thrombocytopenia, the administration schedule of gemcitabine was changed to 3weekly cycles, with the drug administered at the same dose as previously, but on days 1 and 8 , followed by 1 week's rest. From the 40th cycle onward, the administration schedule was changed again to biweekly cycles, with no reduction of the drug dose, because of grade 1 malaise.

After the completion of 5 years of treatment with gemcitabine, we again held discussions with the patient and his family and decided to discontinue the gemcitabine therapy, because the patient had remained relapse free for 45 months. Gemcitabine had been administered a total of 160 times, over 60 cycles, for 5 years, while the drug toxicities had remained rather mild in nature throughout the course of treatment, consisting only of grade 2 leukopenia and thrombocytopenia and grade 1 malaise.

However, 11 months after treatment discontinuation, the patient presented with signs of obstructive jaundice. Brushing cytology of the bile duct by endoscopic retrograde cholangiopancreatography revealed an adenocarcinoma. MRI showed relapse of the tumor at the porta hepatis (fig. 3). The metastases to the lymph nodes invaded the celiac artery and common hepatic artery. Thus, the patient was diagnosed as having unresectable relapse of gallbladder cancer, and gemcitabine therapy was restarted. Six months later, the tumors showed an increase in size and multiple liver metastases appeared. The second term of gemcitabine therapy was less effective against the disease as compared to the first, although the best response was stable disease. At the time this case report was written, the patient was receiving S-1 therapy and has remained alive for 79 months after the commencement of the first gemcitabine therapy. Serial changes of the serum levels of carbohydrate antigen 199 and CEA during the entire treatment are shown in fig. 4.

\section{Discussion}

Gemcitabine is one of the key agents for the treatment of advanced biliary tract cancer, because favorable results of gemcitabine monotherapy for advanced biliary tract cancer have been reported by several other studies. Recently, a randomized phase III trial (ABC-02) showed a significant survival advantage of cisplatin plus gemcitabine over gemcitabine alone in patients with advanced biliary tract cancer [7]. In Japan, similar results to the ABC-02 study were reported from a randomized controlled trial comparing cisplatin plus gemcitabine with gemcitabine alone [8]. Therefore, cisplatin plus gemcitabine has been acknowledged worldwide as the standard treatment for patients with advanced biliary tract cancer. However, our patient reported here had received gemcitabine monotherapy, and not cisplatin plus gemcitabine therapy, for the treatment of advanced gallbladder cancer, because the survival benefit of cisplatin plus gemcitabine had not yet been established at the time of commencement of the chemotherapy in this patient.

There are few reports of CR to chemotherapy in patients with advanced biliary tract cancer. Among them, there are even fewer reports on such an outcome of a case of gallbladder cancer as described here (table 1) [9-13]. Therefore, the outcome of this patient with advanced gallbladder cancer, who showed sustained CR to gemcitabine monotherapy for about 3 years and survived for over 6.5 years after chemotherapy alone, is very meaningful and valuable. 
Suyama et al.: Early Relapse of Unresectable Gallbladder Cancer after Discontinuation of Gemcitabine Monotherapy Administered for 5 Years

How should patients with advanced gallbladder cancer be treated after they have shown CR to chemotherapy? Gemcitabine monotherapy can be continued until disease progression, because there is almost no known cumulative toxicity of gemcitabine. On the other hand, it was generally considered that chemotherapy could be discontinued if there was no tumor relapse. In this patient, chemotherapy was continued until 45 months after CR had been achieved, because the patient and his family were eager to continue with the treatment. We finally decided to discontinue chemotherapy 5 years after the treatment had been commenced, because we were convinced that his tumor had been cured. However, tumor relapse was diagnosed 11 months after the discontinuation of gemcitabine. Why did the tumor relapse even after CR had been sustained for the long period of 45 months? Viable cancer cells resistant to gemcitabine, such as cancer stem cells, might have survived in the gallbladder or lymph nodes for 5 years. Therefore, the second gemcitabine treatment term might not have been as effective against tumor recurrence as the first treatment term. Can unresectable gallbladder cancer not be cured by chemotherapy alone? A complete cure by chemotherapy alone is extremely rare, and most of the patients cannot be cured by chemotherapy alone. Complete surgical resection may need to be attempted even after CR has been achieved with chemotherapy. Finally, for how long should gemcitabine therapy be continued after CR has been confirmed? Would the cancer in this patient not have relapsed if he had continued to receive gemcitabine? There is no consensus yet on the optimal duration of chemotherapy for gallbladder cancer after CR has been achieved. To resolve these issues, accumulation of further cases of CR to therapy will be warranted.

In summary, we report a patient with gallbladder cancer, who after showing CR to gemcitabine monotherapy administered for 5 years, showed early relapse within only 11 months of discontinuation of the treatment. It is necessary to establish a treatment continuation strategy for patients with gallbladder cancer who show CR to gemcitabine therapy.

\section{Acknowledgement}

This work was supported in part by a Grant-in-Aid for Cancer Research and for the Third-Term Comprehensive 10-Year Strategy for Cancer Control from the Ministry of Health, Labour and Welfare of Japan.

\section{Disclosure Statement}

The authors have no conflicts of interest to declare in relation to this paper.

\section{References}

1 Suzuki E, Furuse J, Ikeda M, et al: Treatment efficacy/safety and prognostic factors in patients with advanced biliary tract cancer receiving gemcitabine monotherapy: an analysis of 100 cases. Oncology 2010;79:39-45.

2 Eckel F, Schmid RM: Chemotherapy in advanced biliary tract carcinoma: a pooled analysis of clinical trials. Br J Cancer 2007;96:896-902.

-3 Huang P, Chubb S, Hertel LW, et al: Action of 2',2'-difluorodeoxycytidine on DNA synthesis. Cancer Res 1991;51:6110-6117.

4 Raderer M, Hejna MH, Valencak JB, et al: Two consecutive phase II studies of 5fluorouracil/leucovorin/mitomycin C and of gemcitabine in patients with advanced biliary cancer. Oncology 1999;56:177-180. 


\section{Case Reports in Oncology}

\begin{tabular}{l|l}
\hline Case Rep Oncol 2013;6:531-537 \\
\hline DOI: $10.1159 / 000356080$ & $\begin{array}{l}\text { @ } 2013 \text { S. Karger AG, Basel } \\
\text { www.karger.com/cro }\end{array}$ \\
\hline
\end{tabular}

Suyama et al.: Early Relapse of Unresectable Gallbladder Cancer after Discontinuation of Gemcitabine Monotherapy Administered for 5 Years

5 Okusaka T, Ishii H, Funakoshi A, et al: Phase II study of single-agent gemcitabine in patients with advanced biliary tract cancer. Cancer Chemother Pharmacol 2006;57:647-653.

-6 Kiba T, Nishimura T, Matsumoto S, et al: Single-agent gemcitabine for biliary tract cancers. Study outcomes and systematic review of the literature. Oncology 2006;70:358-365.

7 Valle J, Wasan H, Palmer DH, et al, ABC-02 Trial Investigators: Cisplatin plus gemcitabine versus gemcitabine for biliary tract cancer. N Engl J Med 2010;362:1273-1281.

$\checkmark 8$ Okusaka T, Nakachi K, Fukutomi A, et al: Gemcitabine alone or in combination with cisplatin in patients with biliary tract cancer: a comparative multicentre study in Japan. Br J Cancer 2010;103:469-474.

9 Castro MP: Efficacy of gemcitabine in the treatment of patients with gallbladder carcinoma: a case report. Cancer 1998;82:639-641.

10 Malik IA, Aziz Z, Zaidi SH, et al: Gemcitabine and cisplatin is a highly effective combination chemotherapy in patients with advanced cancer of the gallbladder. Am J Clin Oncol 2003;26:174-177.

11 Doval DC, Sekhon JS, Gupta SK, et al: A phase II study of gemcitabine and cisplatin in chemotherapy-naive, unresectable gall bladder cancer. Br J Cancer 2004;90:1516-1520.

12 Furuse J, Okusaka T, Boku N, et al: S-1 monotherapy as first-line treatment in patients with advanced biliary tract cancer: a multicenter phase II study. Cancer Chemother Pharmacol 2008;62:849-855.

13 Sharma A, Mohanti B, Raina V, et al: A phase II study of gemcitabine and oxaliplatin (Oxigem) in unresectable gall bladder cancer. Cancer Chemother Pharmacol 2010;65:497-502.

Table 1. Reports of patients with gallbladder cancer in whom CR was achieved with chemotherapy

\begin{tabular}{lllll}
\hline Author (year) & Regimen & $\begin{array}{l}\text { Patients in whom } \\
\text { complete response } \\
\text { was achieved, } \mathrm{n} \begin{array}{l}\text { Cycles of } \\
\text { chemotherapy after } \\
\text { complete response } \\
\text { was achieved, } \mathrm{n}\end{array}\end{array}$ & \multicolumn{3}{c}{ Comments } \\
\hline Castro [9] (1998) & Gemcitabine & 1 & 0 & Case report \\
Malik et al. [10] (2003) & Gemcitabine + cisplatin & 1 & 6 & Prospective study of 10 patients \\
Doval et al. [11] (2004) & Gemcitabine + cisplatin & 1 & 18 & Phase II study of 30 patients \\
Furuse et al. [12] (2008) & S-1 & 1 & 6 & Phase II study \\
Sharma et al. [13] (2010) & Gemcitabine + oxaliplatin & 1 & 45 & Phase II study of 48 patients \\
Present case & Gemcitabine & 1 & &
\end{tabular}

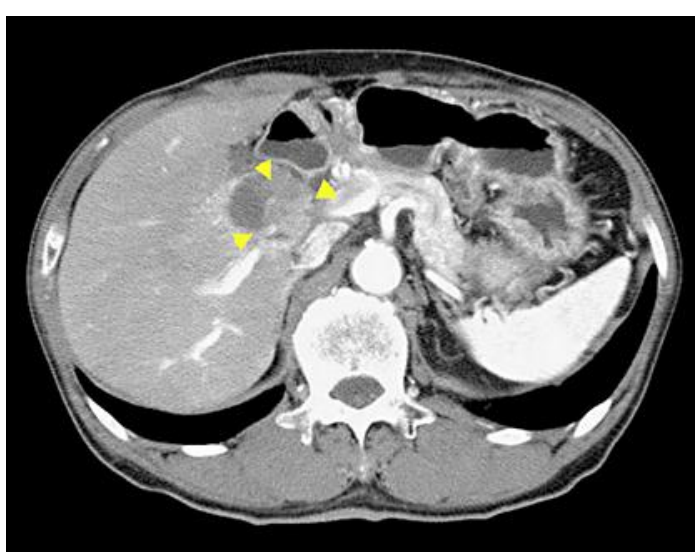

Fig. 1. CT image before the start of gemcitabine therapy. CT revealed a tumor located at the cervix of the gallbladder (arrowheads) also involving the common and right hepatic arteries, and enlargement of the No. 12 lymph node. 


\section{Case Reports in Oncology}

\begin{tabular}{l|l}
\hline Case Rep Oncol 2013;6:531-537 \\
\hline DOI: $10.1159 / 000356080$ & $\begin{array}{l}\text { C 2013 S. Karger AG, Basel } \\
\text { www.karger.com/cro }\end{array}$ \\
\hline
\end{tabular}

Suyama et al.: Early Relapse of Unresectable Gallbladder Cancer after Discontinuation of Gemcitabine Monotherapy Administered for 5 Years
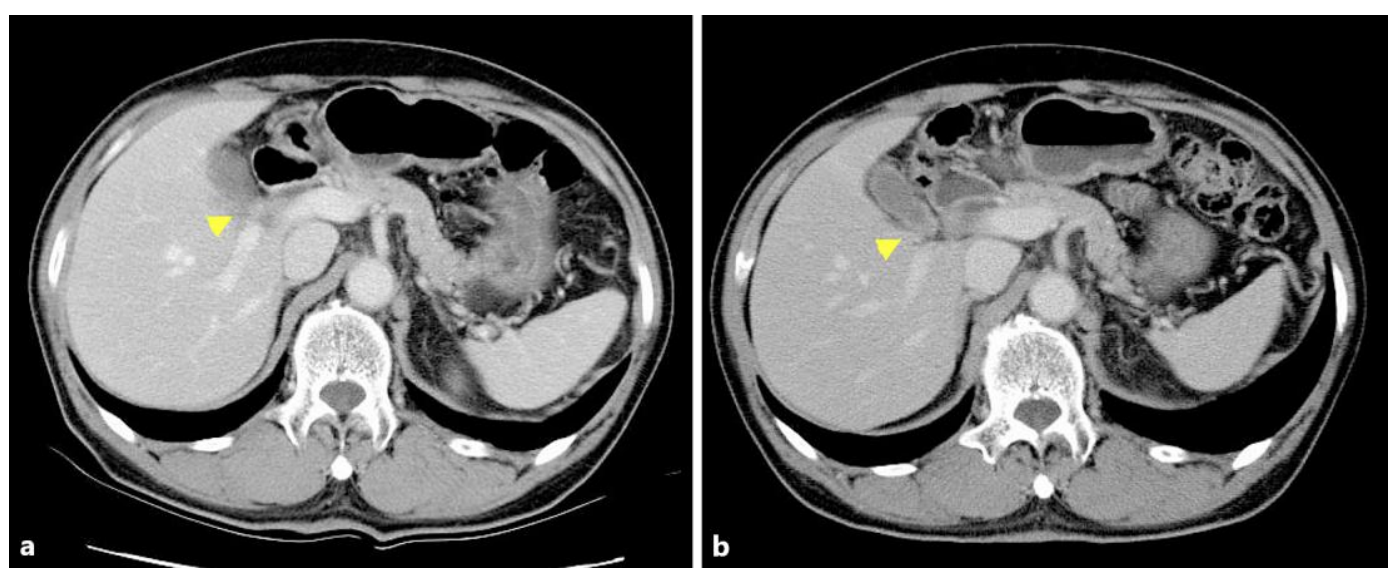

Fig. 2. a CT image obtained after 13 cycles of gemcitabine treatment. The tumor showed a $35 \%$ reduction in diameter, judged as a partial response according to the RECIST criteria, version 1.0. b CT image obtained after 20 cycles of gemcitabine therapy. The tumor has disappeared altogether on the CT images, and CR was confirmed.

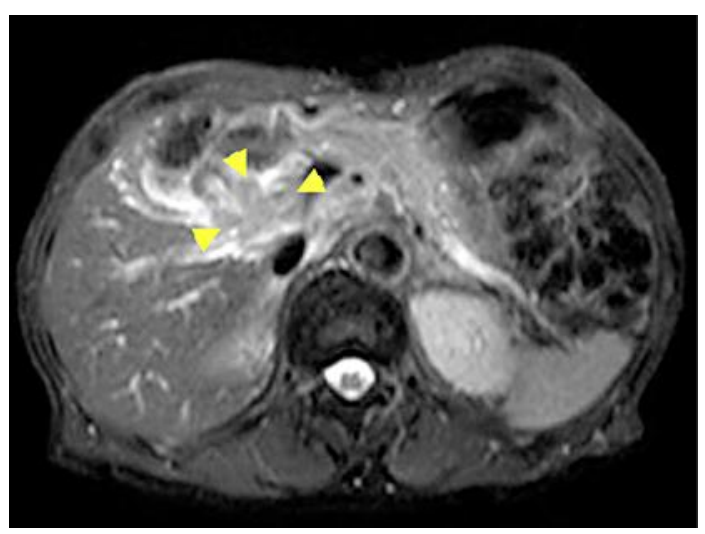

Fig. 3. MRI at the time of the tumor relapse. MRI showed relapse of the tumor at the hilar porta hepatis. The lymph node metastasis invaded the celiac artery and common hepatic artery. 
Suyama et al.: Early Relapse of Unresectable Gallbladder Cancer after Discontinuation of Gemcitabine Monotherapy Administered for 5 Years

\section{CEA (ng/ml)}

CA19-9 (U/ml)

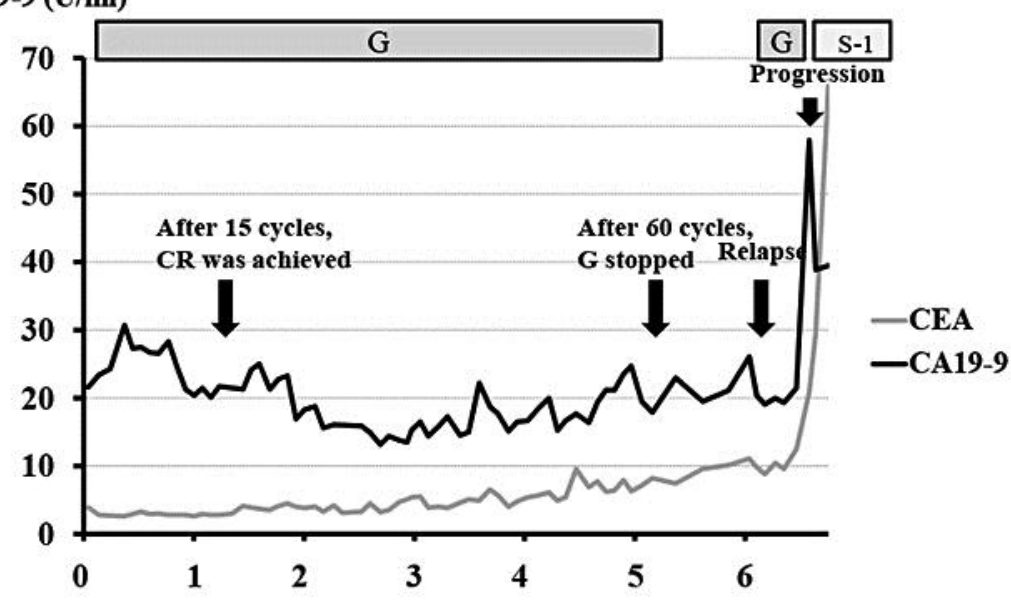

Years after commencement of the first gemcitabine treatment term

Fig. 4. Serial changes of the serum levels of carbohydrate antigen 19-9 and CEA during the treatment course. CA19-9 = Carbohydrate antigen 19-9; G = gemcitabine. 